\title{
Signaling at the Golgi
}

\author{
Peter Mayinger \\ Division of Nephrology and Hypertension and Department of Cell and Developmental Biology, \\ Oregon Health \& Science University, Portland, Oregon 97239 \\ Correspondence: mayinger@ohsu.edu
}

The protein processing and trafficking function of the Golgi is intimately linked to multiple intracellular signaling pathways. Assembly of Golgi trafficking structures and lipid sorting at the Golgi complex is controlled and coordinated by specific phosphoinositide kinases and phosphatases. The intra-Golgi transport machinery is also regulated by kinases belonging to several functionally distinct families, for example, MAP kinase signaling is required for mitotic disassembly of the Golgi. However, the Golgi plays an additional, prominent role in compartmentalizing other signaling cascades that originate at the plasma membrane or at other organelles. This article summarizes recent advances in our understanding of the signaling network that converges at the Golgi.

$T^{\mathrm{h}}$ he Golgi apparatus is a dynamic structure that constantly exchanges proteins and lipids with other organelles. It is critical for organellar homeostasis that the different trafficking routes at the Golgi are precisely regulated. For example, the sorting and transport functions of the Golgi must be correctly coordinated with the overall activity of the secretory pathway. In addition, changes in Golgi structure and morphology are tightly controlled, which is particularly critical during mitosis, when the Golgi complex becomes disassembled for proper distribution between the dividing cells. It is therefore not surprising that diverse sets of signaling factors localize at the Golgi and control its function and shape.

Phosphoinositide lipids have emerged as particularly important regulators of Golgi function. Reversible phosphorylation of the inositol headgroup of phosphatidylinositol creates seven distinct phosphoinositide species (Di Paolo and De Camilli 2006). These molecules serve as signal transducers at virtually every cellular membrane but have a particularly important role in controlling membrane traffic (Di Paolo and De Camilli 2006). A critical property of phosphoinositides is their tightly regulated spatial distribution. Recent studies have uncovered concentrated pools of these lipids at individual membranes including the Golgi (Roy and Levine 2004; De Matteis et al. 2005; Varnai and Balla 2008). Phosphoinositides often act in cooperation with small Ras-type GTPases and the interplay between phosphoinositides and GTPases from the ADP-ribosylation factor (Arf) and Ras-related in brain (Rab) families is essential for Golgi function (Behnia and Munro 2005; Mayinger 2009). How the lipid kinases and phosphatases that regulate Golgi phosphoinositides interact with other

Editors: Graham Warren and James Rothman

Additional Perspectives on The Golgi available at www.cshperspectives.org

Copyright (C) 2011 Cold Spring Harbor Laboratory Press; all rights reserved; doi: 10.1101/cshperspect.a005314

Cite this article as Cold Spring Harb Perspect Biol 2011;3:a005314 
P. Mayinger

signaling pathway remains a challenging area of research.

Whereas phosphoinositide signaling pathways are mainly controlled via extracellular signals that transmit metabolic status and growth conditions, Golgi function can also be regulated by signals that originate at other secretory organelles. Enhanced biosynthesis and processing of secretory proteins at the ER induces the activation of a signaling network that modulates intra-Golgi traffic and overall capacity of secretion (Sallese et al. 2009).

Finally, there is mounting evidence that the Golgi serves as an important signaling platform for numerous signaling cascades that originate at the plasma membrane. The discovery that components of the Ras and the protein kinase A (PKA) pathways reside at the Golgi indicates that this organelle plays an important role in compartmentalizing signal transduction pathways (Quatela and Philips 2006; Sallese et al. 2009). This article will review our current understanding of signaling at the Golgi and also highlight the relevance of these processes for human disease.

\section{CONTROL OF GOLGI FUNCTION BY PHOSPHOINOSITIDES}

Among the seven distinct phosphoinositide species, phosphatidylinositol-4-phosphate (PI(4)P) plays a uniquely important role in regulating Golgi function. PI(4)P displays a high steadystate concentration at the Golgi and the regulation of its spatial distribution is key to the mechanism underlying the organization of the Golgi. (De Matteis et al. 2005; Blagoveshchenskaya et al. 2008; Cheong et al. 2010). The relevance of Golgi PI(4)P for anterograde trafficking was first discovered in the yeast Saccharomyces cerevisiae. Mutations in the PIK1 gene, which encodes the PI 4-kinase responsible for generating Golgi $\mathrm{PI}(4) \mathrm{P}$ in yeast, causes severe defects in the anterograde transport of a variety of cargo proteins (Hama et al. 1999; Walch-Solimena and Novick 1999; Audhya et al. 2000; Schorr et al. 2001). In addition, lipid phosphatases play an important role in maintaining the proper intracellular distribution of PI(4)P (Mayinger 2009).

\section{PI 4-Kinases}

In mammalian cells, both PI4KII $\alpha$ and PI4KIII $\beta$ contribute to the biosynthesis of Golgi PI(4)P (Vicinanza et al. 2008). PI4KII $\alpha$ was also detected at the endoplasmic reticulum (ER), endosomes, and the plasma membrane (Balla et al. 2002; Guo et al. 2003; Minogue et al. 2006). The exact location of PI4KII $\alpha$ and PI4KIII $\beta$ within the Golgi is unclear but functional and localization data indicate that these kinases may generate distinct Golgi pools of $\mathrm{PI}(4) \mathrm{P}$ involved in individual trafficking pathways (Wang et al. 2003; Weixel et al. 2005; Toth et al. 2006).

Although it remains unclear how localization of PI4KII $\alpha$ to the Golgi is regulated, factors controlling the Golgi association of PI4KIII $\beta$ have been characterized. Both, activated Arf1 and the N-myristoylated, $\mathrm{Ca}^{2+}$-binding neuronal calcium sensor-1 (NCS-1) are involved in PI4KIII $\beta$ recruitment to the Golgi (Godi et al. 1999; Zhao et al. 2001; Haynes et al. 2005; de Barry et al. 2006). In yeast, Golgi association of PI 4-kinase Pik1 requires the N-myristoylated cofactor Frq1, which is highly homologous to NCS-1 (Hendricks et al. 1999; Huttner et al. 2003).

PI4KIII $\beta$ and Golgi PI(4)P also play an interesting role in RNA virus replication, which often occurs at specific reorganized intracellular membranes. A new study shows that some viruses can reorganize organelles of the secretory pathway to generate unique membrane compartments for RNA replication (Hsu et al. 2010). Specific viral proteins induce recruitment of PI4KIII $\beta$ to the ER/Golgi intermediate compartment (ERGIC) (Hsu et al. 2010). This regulation requires activation of Arf1 by enteroviral 3A protein, which binds to the Arf1 guanine nucleotide exchange factor GBF1 (Hsu et al. 2010). Enteroviral RNA polymerases specifically bind to $\mathrm{PI}(4) \mathrm{P}$ at the remodeled membranes, which is essential for both enteroviral and flaviviral RNA replication (Hsu et al. 2010). 


\section{PI(4)P Effectors at the Golgi}

$\mathrm{PI}(4) \mathrm{P}$ effectors at the Golgi comprise functionally distinct classes of proteins that control Golgi structure and function (Fig. 1). Golgi $\mathrm{PI}(4) \mathrm{P}$ is important for clathrin-dependent and -independent trafficking pathways that originate at the Golgi (Fig. 1). Both, adaptor protein complex 1 (AP-1) and $\gamma$-ear-containing, ADP-ribosylation factor-binding proteins (GGAs) bind simultaneously to PI(4)P and to activated Arf1 (Heldwein et al. 2004; Wang et al. 2007; Demmel et al. 2008b). This coincidence detection mechanism controls the formation of clathrin-coated vesicles at the transGolgi network (TGN) and may be involved in distinct trafficking pathways via late or early endosomal compartments (Abazeed and Fuller 2008).

Recent evidence in yeast suggests that Golgi $\mathrm{PI}(4) \mathrm{P}$ is also important for regulating the activity of Rab GTPases (Fig. 1). The Rab GEF Sec2 is recruited to the TGN by simultaneously binding to PI(4)P and the activated Rab GTPase Ypt32 (Mizuno-Yamasaki et al. 2010). PI(4)P thus acts in parallel with Ypt32 in a coincidence detection mechanism that controls Sec 2 recruitment to the Golgi and in turn prevents Sec2 association with the exocyst. Once a trafficking vesicle containing Sec2 and Ypt32 buds off the TGN, the downstream-acting Rab Sec4 replaces Ypt32 and is activated by Sec2, which leads to recruitment of Sec15 and other components of the exocyst tethering complex (MizunoYamasaki et al. 2010). Consequently, the interaction between PI(4)P and Sec2 at the TGN regulates the proper timing of Sec4 activation, which can only occur at post-Golgi vesicles that contain little or no PI(4)P. This precise temporal control of Rab protein activation is intimately linked to transport vesicle maturation.

Several lipid transfer proteins bind to Golgi PI(4)P (Fig. 1). Ceramide transfer protein (CERT), oxysterol-binding proteins (OSBPs), and four-phosphate adaptor protein 1 and 2 (FAPP1, 2) contain PH-domains that specifically recognize PI(4)P and activated Arf1. Both CERT and FAPP2 are involved in sphingolipid biosynthesis. Ceramide is synthesized at the ER and transferred to the Golgi by CERT where it is converted to both sphingomyelin and

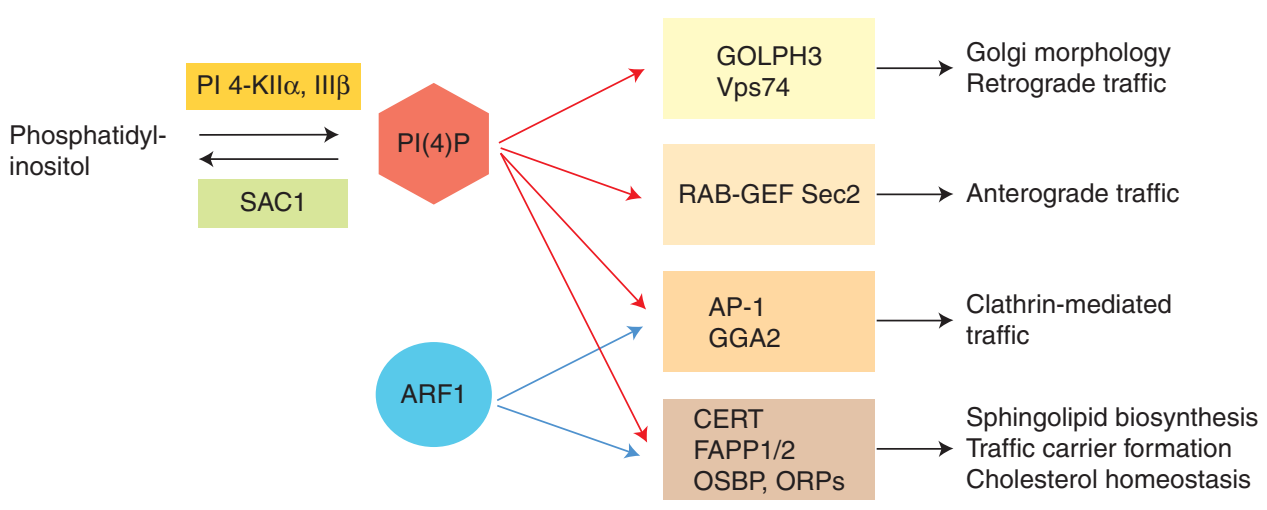

Figure 1. PI(4)P effectors at the Golgi. Several functionally distinct types of PI(4)P effectors are recruited to the Golgi. In many cases, recruitment to the Golgi also requires activated Arf1, which together with PI(4)P functions in a coincidence-detection mechanism that provides additional specificity. Ras-GEF Sec2 regulates Rab function in anterograde trafficking from the Golgi, whereas the clathrin adaptors AP-1 and the GGA proteins function in clathrin-mediated sorting to the endosomal/lysosomal system. Another group of effectors consists of the lipid transfer proteins CERT, FAPP1/2, OSBP, and OSBP-related proteins (ORPs), which are important for nonvesicular transfer of sterols, ceramide, and glucosylcermaide. FAPP2 plays a dual role in glycosphingolipid biosynthesis and in membrane tubule formation. GOLPH3 and its yeast homologue Vps74 play a role in Golgi structure and in the proper localization of glycosylation enzymes. 
P. Mayinger

glucosylceramide (Yasuda et al. 2001). FAPP2 was discovered independently as a factor required for glycosphingolipid biosynthesis and for anterograde trafficking from the Golgi and its physiological role remains somewhat controversial (Godi et al. 2004; Vieira et al. 2005; D'Angelo et al. 2007; Halter et al. 2007). Recent reports show that FAPP2 is required for the delivery of glucosylceramide to appropriate sites for the biosynthesis of complex glycosphingolipids. (D'Angelo et al. 2007; Halter et al. 2007). However, there is also evidence that FAPP2 can form dimers that induce the tubulation of membranes in vitro (Cao et al. 2009). Tubulation activity is dependent on phosphoinositide-binding and the wedgelike structure of the FAPP2 PH-domain (Cao et al. 2009; Lenoir et al. 2010). FAPP2 may therefore couple the synthesis of glycosphingolipids with the formation of tubular trafficking carriers required for the export of proteins and lipids to the cell surface. (Cao et al. 2009; Yui et al. 2009; Lenoir et al. 2010). OSBP and OSBP-related proteins (ORPs) also bind to Golgi PI(4)P and Arf1. The exact cellular function of these proteins is not entirely clear. There is evidence that these proteins catalyze the nonvesicular sterol transfer between closely apposed membranes, such as at organelle contact sites (Schulz et al. 2009). However, these proteins may also act as cholesterols sensors required for the regulation of vesicular traffic (Li et al. 2002; Kozminski et al. 2006). Recent studies indicate that CERT, OSPB, and specific OSBP-related proteins (ORPs) may coordinate nonvesicular sterol and ceramide transport between ER and Golgi (Peretti et al. 2008; Ngo and Ridgway 2009). Together, these studies show that secretory proteins and lipids can use different transport pathways to transit the Golgi. However, how nonvesicular lipid transfer between ER and Golgi or within the Golgi is linked functionally to membrane trafficking of secretory proteins from the TGN remains unclear. It is possible that coordinated enrichment of sphingolipids and sterols at the TGN is required to produce transport carriers for the export of secretory proteins. Such a mechanism could be important for maintaining the unique lipid composition of the plasma membrane.

Finally, Golgi phosphoprotein 3 (GOLPH 3) represents a new class of proteins that is recruited to the Golgi by PI(4)P (Fig. 1). GOLPH3 binds also the unconventional myosin MYO18A and this interaction appears to be necessary for normal Golgi trafficking and morphology (Dippold et al. 2009). Interestingly, the GOLPH3 gene is located on human chromosome $5 \mathrm{p} 13$, which is frequently amplified in multiple solid tumor types (Scott et al. 2009). Recent functional analyses show that GOLPH3 can function as an oncoprotein to promote cell transformation and tumor growth. GOLPH3 stimulates the activity of the mammalian target of rapamycin (mTOR), a serine/ threonine kinase involved in the regulation of cell growth and survival (Scott et al. 2009). Yeast Vps74, an orthologue of human GOLPH3, also binds specifically to Golgi PI(4)P (Wood et al. 2009). Vps74 interacts with the cytosolic domains of cis and medial Golgi glycosylation enzymes and also with COP-I and it was proposed that Vps74 may function in the steadystate localization of Golgi glycosyltransferases by promoting their incorporation into retrograde COPI-coated vesicles (Schmitz et al. 2008; Tu et al. 2008); (A detailed review of Golgi enzyme localization is provided in Banfield 2011). Although the precise mode of action of GOLPH3/Vps74 remains to be elucidated, this factor represents an intriguing mechanistic link between phosphoinositide signaling, Golgi glycosylation and the regulation of cell size and growth-factor-induced mTOR signaling in human cells, which appears to be highly relevant for tumorigenesis.

\section{Golgi PI(4)P and Cell Growth}

During cell growth, the plasma membrane expands and lipids and proteins must be delivered to the cell surface via the secretory pathway. Consequently, a crosstalk between phosphoinositide signaling at the Golgi and cell growth is required to adapt the secretory capacity of proliferating cells with their growth rates. A functional secretory pathway is essential for 
normal cell viability but this pathway is often up-regulated in malignant tumors and may be linked to rapid cancer cell growth (He et al. 2002; Cheng et al. 2005).

Recent evidence suggests that the lipid phosphatase Sacl is a key regulator of Golgi PI(4)P during cell growth (Fig. 2A,B). Although Sac1 can dephosphorylate several phosphoinositides, PI(4)P appears to be the most important substrate in vivo. In yeast, Sacl forms a complex with Dpm1 at the ER (Fig. 2A), which is important for oligosaccharide biosynthesis (Faulhammer et al. 2005). Dpm1 synthesizes dolicholphosphate-mannose, an essential mannosyl donor for $\mathrm{N}$ - and O-linked glycosylation pathways and for the biosynthesis of the glycosylphosphatidylinositol (GPI) anchor at the ER. The formation of a Sacl and Dpm1 complex depends on the metabolic status of yeast cells. As long as glucose is not limiting, the complex with Dpm1 persists and localization of Sacl is restricted to ER membranes (Faulhammer et al. 2005; Faulhammer et al. 2007). Yeast Sac1 is also associated with serine palmitoyltransferase at ER membranes and may be involved in coordinating sphingolipid biosynthesis and trafficking (Breslow et al. 2010; Han et al. 2010). When yeast cells are starved for glucose, Sacl translocates rapidly to the Golgi (Faulhammer et al. 2005) (Fig. 2A). Interestingly, Golgi association of PI-4 kinase Pik1 is regulated in the opposite manner (Fig. 2A). During exponential cell growth, when glucose is abundant, Pik1 localizes to

A Yeast

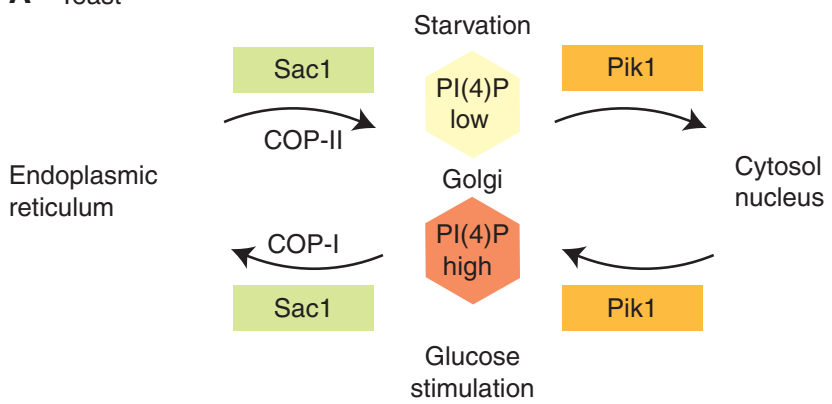

B Mammals

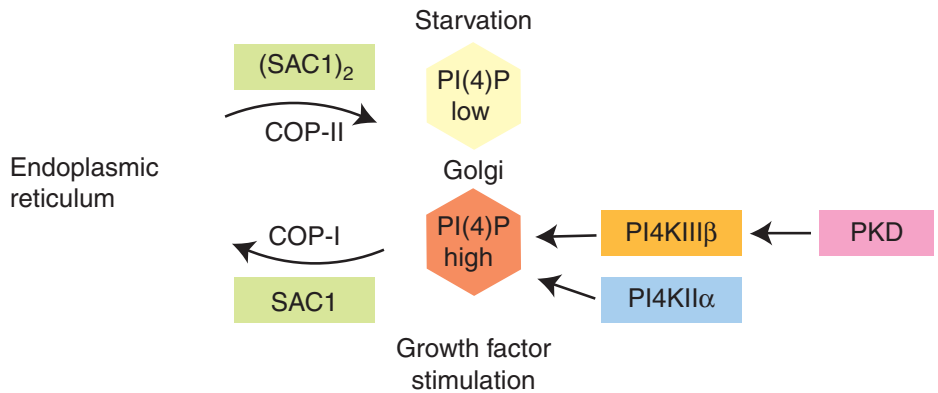

Figure 2. Control of Golgi PI(4)P during cell growth. (A): Regulation of Golgi PI(4)P in yeast. Metabolic control of PI(4)P in yeast involves translocation of the lipid phosphatase Sac1 between Golgi and ER and requires Golgi-recruitment of the lipid kinase Pik1. Golgi association of Sacl and Pik1 are controlled in an opposite manner by glucose. (B): Regulation of Golgi PI(4)P in mammals. In the presence of growth factors, SAC1 lipid phosphatase is largely localized to the ER and Golgi and PI(4)P is synthesized by PI4KII $\alpha$ and PI4KIII $\beta$. PI4KIII $\beta$ is activated by PKD. During serum starvation, SAC1 forms oligomers, translocates to the Golgi and downregulates Golgi $\mathrm{PI}(4) \mathrm{P}$. 
P. Mayinger

the Golgi, where it generates high steady state levels of PI(4)P (Faulhammer et al. 2007; Demmel et al. 2008a). Pik1 is in a complex with Frq1, an $\mathrm{N}$-myristoylated cofactor that functions in Golgi membrane targeting (Hendricks et al. 1999). During glucose starvation, the Pik1/Frq1 complex is released from the Golgi. A certain proportion of Pik1p also localizes to the nucleus, where it has an essential but not well-understood function (Strahl et al. 2005). The exact distribution of Pik1 between Golgi, nucleus, and cytoplasm at different growth conditions appears to involve binding to 14-3-3 proteins (Demmel et al. 2008a). During exponential growth, the mutually exclusive localization of Sac1 and Pik1 produces a Golgi-restricted pool of PI(4)P, which is essential for efficient anterograde trafficking (Faulhammer et al. 2005; Faulhammer et al. 2007). This regulation of Golgi PI(4)P is therefore part of a metabolic control mechanism that coordinates the secretory capacities of ER and Golgi with the rate of cell growth and proliferation.

The physiological role of Sac1 in the regulation of Golgi PI(4)P during growth is evolutionary conserved. In contrast to yeast cells, however, localization of human SAC1 responds to growth factors and not to nutrients (Fig. 2B). Human SAC1 becomes concentrated at the Golgi following serum starvation and downregulates Golgi $\mathrm{PI}(4) \mathrm{P}$ but rapidly translocates to the ER when cell growth is stimulated with growth factors (Blagoveshchenskaya et al. 2008) (Fig. 2B). Reversible oligomerization of $\mathrm{SACl}$, which is regulated by growth factor signaling, determines recruitment into coat protein complex II (COP-II) or coatomer protein complex I (COP-I) vesicles, respectively, (Blagoveshchenskaya et al. 2008).

A certain proportion of SAC1 remains at the cisternal Golgi after growth stimulation. This portion of SAC1 is essential for controlling the spatial distribution of $\mathrm{PI}(4) \mathrm{P}$ within the Golgi in proliferating cells, resulting in concentration of PI(4)P at the TGN (Cheong et al. 2010). Depletion of SAC1 causes accumulation of PI(4)P at other intracellular membranes and leads to peripheral mislocalization of Golgi glycosylation enzymes (Cheong et al. 2010). SAC1-dependent restriction of PI(4)P to the TGN may be important for preventing anterograde traffic of Golgi enzymes to the cell periphery. Because retrograde traffic of glycosylation enzymes in yeast requires PI(4)P-binding effectors such as Vps74 (Wood et al. 2009), it is also possible that COP-I-mediated retrograde transport of Golgi enzymes is impaired in SAC1-depleted cells.

Rapid translocation of SAC1 from the Golgi back to the ER after growth factor stimulation requires the p38 mitogen-activated protein kinase (MAPK) (Blagoveshchenskaya et al. 2008). Thus, SAC1 may be a downstream p38 MAPK effector that modulates growth factor induced cell proliferation by controlling Golgi function. However, it remains to be determined whether p38 interacts with SAC1 directly or with factors that control SAC1 localization.

\section{PKC AND PKD SIGNALING AND GOLGI FUNCTION}

Several Protein Kinase C (PKC) and Protein Kinase D (PKD) isoforms localize to the Golgi complex. This interaction depends on Golgi diacylglycerol (DAG), which is recognized by the C1b domain of PKC (Schultz et al. 2004). PKD1 and PKD2 also bind specifically to DAG and are recruited to the Golgi (Maeda et al. 2001). Activation of phospholipase D (PLD) by Arf1 at the Golgi generates phosphatidic acid, which in turn is converted to DAG by phosphatidic acid phosphohydrolase (Riebeling et al. 2009). This pathway for DAG biosynthesis seems to be physiologically relevant because ilimaquinone, a drug triggering Golgi fragmentation, requires PLD to stimulate PKD activity (Sonoda et al. 2007). However, DAG is also the byproduct of sphingomyelin biosynthesis at the Golgi. Sphingomyelin synthases transfer a phosphocholine from phosphatidylcholine to ceramide thus generating DAG. Golgi DAG is therefore modulated by sphingomyelin synthases SMS1 and SMS2 and it was shown that down-regulation of SMS1 and SMS2 reduce the localization of PKD to the Golgi (Villani et al. 2008). 
Ceramide-induced apoptosis involves translocation and activation of protein kinase $\mathrm{C}-\delta$ and $-\theta$ at the Golgi complex (Kajimoto et al. 2001; Schultz et al. 2003; Kajimoto et al. 2004). In contrast, PKC- $\eta$ is specifically required to drive a regulatory network that controls generation of transport carriers at the TGN. This mechanism involves activation of a heterotrimeric GTP-binding protein at the Golgi resulting in up-regulation of DAG via the subunits $\beta \gamma$ (Diaz Anel and Malhotra 2005). The increase in DAG and interaction with $\beta \gamma$-subunits triggers recruitment of $\mathrm{PKD}$ (Diaz Anel and Malhotra 2005). PKC- $\eta$ then activates PKD at the TGN (Diaz Anel and Malhotra 2005; Bossard et al. 2007). Expression of constitutively activated PKC- $\eta$ or PKD induces Golgi fragmentation, probably because it causes abnormal or enhanced fission events (Diaz Anel and Malhotra 2005; Bossard et al. 2007); (a detailed review of PKD function at the TGN is provided in Malhotra and Campelo 2010). Nocodazole-induced Golgi fragmentation is also associated with selective activation of Golgi-localized PKD (Fuchs et al. 2009).

PI4KIII $\beta$ is another target of protein kinase D (PKD) (Fig. 3). PI4KIII $\beta$ can be phosphorylated by PKD1 and PKD2 at a specific site, which induces binding of 14-3-3 proteins and stabilizes the activated form of PI4KIII $\beta$ (Hausser et al. 2005; Hausser et al. 2006). It is therefore possible that activation of PI4KIII $\beta$ contributes to the PKD-dependent mechanism of transport carrier formation. PI4KIII $\beta$ activation by PKD is also important for the PI(4)Pdependent interaction of the ceramide transfer protein CERT with the Golgi (Fig. 3). CERT binds sequentially to VAP proteins at the ER and to PI(4)P at the Golgi, which results in vectorial transfer of ceramide from the ER to the Golgi (Hanada et al. 2003; Kawano et al. 2006; Lev et al. 2008; Yamaji et al. 2008). At the Golgi, CERT is phosphorylated by PKD, which decreases its binding affinity toward PI(4)P thus triggering release from the Golgi and translocation to the ER (Fugmann et al. 2007) (Fig 3). At the ER, CERT binds to VAP-A and is dephosphorylated by protein phosphatase $2 \mathrm{C}-\varepsilon$ (PP2C- $\varepsilon$ ), which in turn causes release of CERT from the ER and redistribution to the Golgi (Fig. 3) (Hanada et al. 2007; Saito et al. 2008). Thus, PKD is intimately involved in ceramide transfer to the Golgi and functions as multi-step regulator in this mechanism.

\section{RAS/MAP KINASE SIGNALING AT THE GOLGI}

Ras GTPases act as signaling factors downstream from mitogen receptors at the plasma membrane in response to activation by extracellular stimuli. Activated Ras regulates several downstream effectors and is thus involved in controlling multiple steps in cell growth, proliferation, differentiation, and apoptosis. Although it was initially believed that Ras

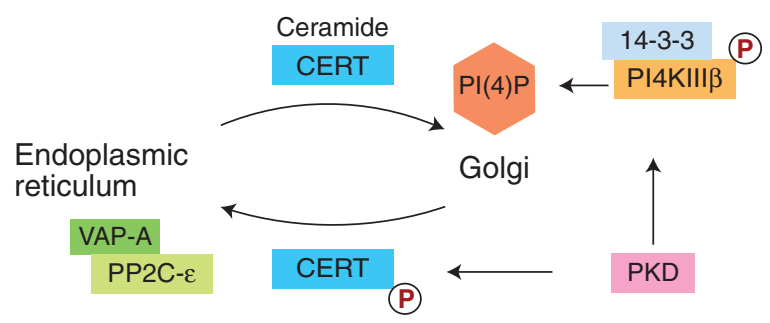

Figure 3. Control of ceramide transfer by PKD. PKD plays sequential roles in ceramide transfer from the ER to the Golgi. PKD stimulates PI(4)P synthesis by activating PI4KIII $\beta$. PI(4)P is essential for Golgi targeting of the ceramide transfer protein CERT. Golgi-localized CERT is also phosphorylated by PKD, which reduces its affinity to $\mathrm{PI}(4) \mathrm{P}$ triggering dissociation from the Golgi and binding to VAP-A at the ER. VAP-A forms a ternary complex with CERT and protein phosphatase $2 \mathrm{C}-\varepsilon(\mathrm{PP} 2 \mathrm{C}-\varepsilon)$, which is required for dephosphorylation and release of CERT. 
P. Mayinger

signaling is confined to the plasma membrane, it has now been recognized that Ras proteins translocate between the plasma membrane and various intracellular organelles (Quatela and Philips 2006). Localization to distinct intracellular membranes is required for Ras protein biogenesis. For their interaction with membranes, Ras proteins require specific posttranslational modifications with lipid anchors, which occur at various compartments including the ER and Golgi (Quatela and Philips 2006). Ras proteins contain a conserved CAAX motif at their carboxy-termini, which is prenylated in the cytosol by farnesyl transferase (Casey and Seabra 1996). Subsequent AAX proteolysis and carboxymethylation of the carboxy-terminal prenylcysteine takes place at the ER (Dai et al. 1998; Schmidt et al. 1998). N-Ras, H-Ras, and K-Ras4A are then further modified by Golgi-localized palmitoyltransferases, whereas K-RasB is not palmitoylated (Swarthout et al. 2005).

A rapid de- and repalmitoylation cycle and translocation between plasma membrane and the Golgi is required for regulating the subcellular distribution of certain Ras isoforms (Goodwin et al. 2005; Rocks et al. 2005). In addition, palmitoylated Ras can be released from the plasma membrane following stimulation and translocate back to the Golgi (Fivaz and Meyer 2005). However, Ras signaling at the Golgi does not require retrograde trafficking and Ras activation can occur directly at the Golgi (Chiu et al. 2002). Specific Golgi-localized GEFs have been detected that play a role in regulating local Ras activity (Fivaz and Meyer 2005). Src-dependent activation of phospholipase C- $\gamma 1$ triggers an increase in Golgi DAG resulting in translocation of the GEF RasGRP1 to the Golgi where it activates Ras (Bivona et al. 2003). RasGRP1 belongs to a family of $\mathrm{C} 1$ domain-containing Ras GEFs that are additionally stimulated by $\mathrm{Ca}^{2+}$ but binding to DAG is essential for Golgi recruitment (Bivona et al. 2003; Zhang et al. 2009). Golgi-localized activation of Ras is particularly important for T-cell development and function. Low-grade stimulation of the T-cell receptor activates specifically N-Ras, which occurs exclusively at the Golgi apparatus (Perez de Castro et al. 2004). Selective Golgi activation of N-Ras occurs also via an endothelial nitric oxide synthase (eNOS)-specific pathway in $\mathrm{T}$ cells interacting with antigen presenting cells. This mechanism involves eNOS-dependent S-nitrosylation of N-Ras and it is possible that eNOS-derived NO directly activates N-Ras at the Golgi (Ibiza et al. 2008). The physiological relevance of Golgi versus plasma membrane activation of Ras in lymphocytes is not entirely clear, but it is possible that the localization of Ras activation at the Golgi induces a more sustained activation of the downstream kinases V-raf-1 murine leukemia viral oncogene homolog (Raf) and extracellular signal-regulated kinase (ERK) compared to activation at the plasma membrane, which is more transient (Yasuda and Kurosaki 2008). There is also evidence for additional downstream effectors of Ras at the Golgi. For example, PLC- $\varepsilon$ contains a Ras-binding domain and is recruited by activated Ras to the plasma membrane and to the Golgi apparatus (Edamatsu et al. 2006). The recently identified protein RAIN is specifically recruited to the Golgi by Ras and may represent another Ras effector at the Golgi but its cellular function remains unclear (Mitin et al. 2004).

Localization to the Golgi of specific Ras effectors can also lead to down-regulation of the Ras-Raf-ERK pathway. The recently discovered protein "Raf kinase trapping to Golgi" (RKTG) is a negative regulator of ERK signaling that functions by sequestering Raf1 and B-Raf to the Golgi, which in turn blocks downstream signaling (Feng et al. 2007; Fan et al. 2008). Another negative regulator of Ras-Raf-ERK signaling is the product of Retinoid-inducible gene 1 (RIG1). The RIG1 protein is localized at the ER and the Golgi, where it forms a complex with Ras and thus inhibits Ras activation (Tsai et al. 2006).

Although the Golgi is an important platform for integrating different aspects of RasRaf-ERK signaling, MAP kinases also play an important role in regulating the structure and function of the Golgi itself. ERK MAP kinases regulate the polarization of Golgi structures in migrating cells (Bisel et al. 2008) and promote 
Golgi disassembly during mitosis, which is reviewed Wang and Seemann 2011. In a recent RNA interference screen, over 100 kinases and phosphatases were identified that affect the ER or the Golgi. Among the identified kinases was ERK2 that targets Sec16 and thus controls coat protein II (COP-II) vesicle biogenesis at ER exit sites (Farhan et al. 2010).

\section{CYCLIC AMP/PROTEIN KINASE A-MEDIATED CONTROL OF GOLGI FUNCTION}

The cAMP/PKA pathway is a conserved signaling pathway that is spatially and temporally controlled to attain tight specificity. It is well established that components of this pathway are present at the Golgi (Cheng and Farquhar 1976; Nigg et al. 1985). A-kinase anchoring proteins (AKAPs) bind to the regulatory subunits of the tetrameric PKA holoenzyme and recruit PKA to specific intracellular compartment including the Golgi (Shanks et al. 2002; Li et al. 2003). PKA is then activated following cAMP-binding, which triggers dissociation of the catalytic and regulatory subunits. The Golgi serves as an important platform for PKA signaling. A certain population of the PKA holoenzyme is concentrated at the Golgi complex (Martin et al. 1999). Following stimulation of adenylate cyclase, the dimeric catalytic subunits dissociate from the Golgi and accumulate in the nucleus, whereas the regulatory subunits remain associated with the Golgi complex (Nigg et al. 1985). It is therefore possible that the Golgi can function as a relay for integrating signaling events originating at the plasma membrane, such as G-protein-coupled receptor activation, with transcriptional regulation in the nucleus.

In addition, PKA is directly involved in controlling multiple aspects of Golgi trafficking and morphology (Fig. 4). An increase in the level of intracellular cAMP induces redistribution of Arf1 from the cytosol to the Golgi (Martin et al. 2000). Recombinant PKA catalytic subunits or cAMP stimulate binding of Arf1 to isolated Golgi membranes and this in vitro membrane recruitment of Arf1 is prevented by specific PKA inhibitors (Martin et al. 2000). These results suggest that PKA may be a regulator of COP-I specific trafficking at the Golgi. Another target of PKA is the KDEL receptor (KDEL-R) (Fig. 4). PKA-dependent phosphorylation at its carboxy-terminal tail is required for retrograde transport of the KDEL receptor from the Golgi to the ER (Cabrera et al. 2003). The KDEL receptor is a transmembrane protein activated by binding to KDEL motifcontaining proteins at the Golgi, which induces dimerization, COP-I complex recruitment and retrograde trafficking to the ER (Pelham 1991).

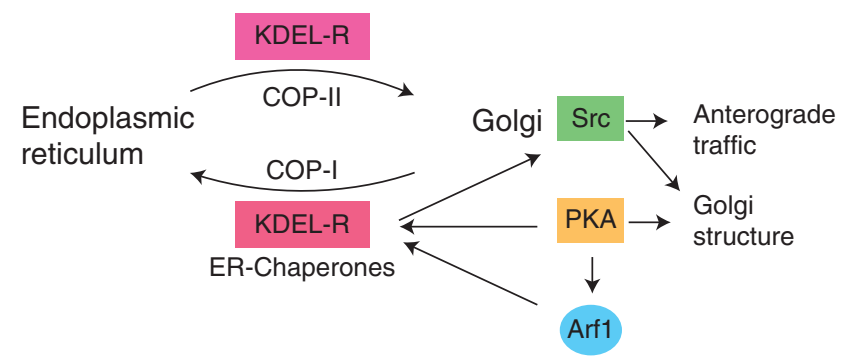

Figure 4. Control of Golgi function by PKA and Src family kinase. A certain sub-population of PKA holoenzyme is localized at the Golgi, where it can be activated by increases in intracellular cAMP. Activated PKA phosphorylates Arf1 and KDEL receptor, which is important for retrograde recycling of ER proteins from the cis-Golgi. KDEL receptor in complex with ER chaperones activates Golgi-localized Src family kinases (Src), which in turn up-regulates intra-Golgi traffic. In addition, activation of Src induces changes in Golgi structure and induces vesiculation during secretion. PKA is also involved in regulating Golgi structure but the specific targets in this mechanism are unknown. 
P. Mayinger

This process is important for recycling ER resident proteins that continuously escape to Golgi compartments (Pelham 1991). The signaling adaptor Cbl localizes to the Golgi in a molecular complex that also contains activated Src kinase (Bard and Baron 1997). A recent study now shows that binding of KDEL receptor to ER chaperones activates Golgi-localized Src kinases, which in turn up-regulates Golgi trafficking and secretory capacity (Pulvirenti et al. 2008) (Fig. 4). The GTPase dynamin 2 (Dyn2) is phosphorylated by Src when a high load of secretory cargo passes through the TGN (Weller et al. 2010). Activation of Src leads to the vesiculation of Golgi cisternae, which is Dyn2 dependent (Weller et al. 2010). Dyn2 is therefore an important Src effector that regulates Golgi function and morphology during active secretion.

PKA is also involved in another aspect of anterograde transport. In hepatocytes, PKA activity is required for efficient trafficking of apical proteins and lipids (Zegers and Hoekstra 1997; Wojtal et al. 2006). Displacement of PKA-RII $\alpha$ from the Golgi apparatus inhibits anterograde transport of MDR1 P-glycoprotein and of glycosphingolipids from the Golgi to apical canalicular membranes (Wojtal et al. 2006). However, PKA-RII displacement from the Golgi does not abolish other aspects of cAMP mediated apical plasma membrane biogenesis in hepatocytes and it is possible that additional cAMP effectors may play a role in canalicular development (Wojtal et al. 2006). In addition, protein kinase $\mathrm{A}$ activity is required for constitutive transport from the TGN to the plasma membrane in nonpolarized cells, and an increase in cAMP synthesis accelerates the rate of anterograde traffic (Muniz et al. 1996; Muniz et al. 1997).

Finally, Golgi morphology and biogenesis depend on PKA activity. Depletion of PKA regulatory subunits using RNAi or inhibition of PKA with specific drugs induces fragmentation of the Golgi (Bejarano et al. 2006). Conversely, stimulation with cAMP triggers fusion and condensation of individual Golgi cisternae to more compact structures (Mavillard et al. 2010). As a consequence, passage of secretory proteins through the Golgi stacks is increased and the efficiency of glycan processing slightly decreased (Mavillard et al. 2010). These observations indicate that resident PKA activity at the Golgi is involved in regulating Golgi structure and function in response to extracellular signals.

\section{CONCLUDING REMARKS}

The Golgi complex is both target and relay station for multiple intracellular signaling pathways and phosphoinositides have emerged as key regulators. Growth-dependent regulation of Golgi PI(4)P is an important and novel mechanism for synchronizing secretion and cell proliferation. The identification of distinct PI(4)P effectors at the Golgi has added important insights into the coordination of sphingolipid biosynthesis, anterograde Golgi traffic and Golgi morphology. The Golgi is also controlled by multiple protein kinase pathways that organize Golgi traffic and shape. Much remains to be learned as to how these diverse pathways are activated at the Golgi and how they contribute to the global control of secretion. The recent discovery of localized activation of Ras and MAP kinase pathways at the Golgi has added an important additional dimension to the field of compartmentalized signaling. Deciphering the exact role of the Golgi in controlling and connecting these pathways will be key in understanding the temporal and spatial integration of intracellular signaling networks.

\section{ACKNOWLEDGMENT}

The research of Peter Mayinger is supported by grants from the National Institutes of Health/ NIGMS (R01GM071569; R01GM084088).

\section{REFERENCES}

Abazeed ME, Fuller RS. 2008. Yeast Golgi-localized,
y-Ear-containing, ADP-ribosylation factor-binding pro-
teins are but adaptor protein-1 is not required for cell-free
transport of membrane proteins from the trans-Golgi
network to the prevacuolar compartment. Mol Biol Cell
19: $4826-4836$.
Audhya A, Foti M, Emr SD. 2000. Distinct roles for the
yeast phosphatidylinositol 4-kinases, Stt4p and Piklp, 
in secretion, cell growth, and organelle membrane dynamics. Mol Biol Cell 11: 2673-2689.

Balla A, Tuymetova G, Barshishat M, Geiszt M, Balla T. 2002. Characterization of type II phosphatidylinositol 4-kinase isoforms reveals association of the enzymes with endosomal vesicular compartments. J Biol Chem 277: 20041-20050.

Banfield DK. 2011. Mechanisms of protein retention in the golgi. Cold Spring Harb Perspect Biol doi:10.1101/ cshperspect.a005264.

Bard F, Baron R. 1997. C-cbl and c-src are associated with the Golgi-apparatus. Mol Biol Cell 8: 758-758.

Behnia R, Munro S. 2005. Organelle identity and the signposts for membrane traffic. Nature 438: 597-604.

Bejarano E, Cabrera M, Vega L, Hidalgo J, Velasco A. 2006. Golgi structural stability and biogenesis depend on associated PKA activity. J Cell Sci 119: 3764-3775.

Bisel B, Wang Y, Wei JH, Xiang Y, Tang D, Miron-Mendoza M, Yoshimura S, Nakamura N, Seemann J. 2008. ERK regulates Golgi and centrosome orientation towards the leading edge through GRASP65. J Cell Biol 182: 837-843.

Bivona TG, Perez De Castro I, Ahearn IM, Grana TM, Chiu VK, Lockyer PJ, Cullen PJ, Pellicer A, Cox AD, Philips MR. 2003. Phospholipase $\mathrm{C} \gamma$ activates Ras on the Golgi apparatus by means of RasGRP1. Nature 424: 694-698.

Blagoveshchenskaya A, Cheong FY, Rohde HM, Glover G, Knodler A, Nicolson T, 89Boehmelt G, Mayinger P. 2008. Integration of Golgi trafficking and growth factor signaling by the lipid phosphatase SAC1. J Cell Biol 180: 803-812.

Bossard C, Bresson D, Polishchuk RS, Malhotra V. 2007. Dimeric PKD regulates membrane fission to form transport carriers at the TGN. J Cell Biol 179: 1123-1131.

Breslow DK, Collins SR, Bodenmiller B, Aebersold R, Simons K, Shevchenko A, Ejsing CS, Weissman JS. 2010. Orm family proteins mediate sphingolipid homeostasis. Nature 463: 1048-1053.

Cabrera M, Muniz M, Hidalgo J, Vega L, Martin ME, Velasco A. 2003. The retrieval function of the KDEL receptor requires PKA phosphorylation of its C-terminus. $\mathrm{Mol}$ Biol Cell 14: 4114-4125.

Cao X, Coskun U, Rossle M, Buschhorn SB, Grzybek M, Dafforn TR, Lenoir M, Overduin M, Simons K. 2009. Golgi protein FAPP2 tubulates membranes. Proc Natl Acad Sci 106: 21121-21125.

Casey PJ, Seabra MC. 1996. Protein prenyltransferases. J Biol Chem 271: 5289-5292.

Cheng H, Farquhar MG. 1976. Presence of adenylate cyclase activity in Golgi and other fractions from rat liver. II. Cytochemical localization within Golgi and ER membranes. J Cell Biol 70: 671-684.

Cheng KW, Lahad JP, Gray JW, Mills GB. 2005. Emerging role of RAB GTPases in cancer and human disease. Cancer Res 65: 2516-2519.

Cheong FY, Sharma V, Blagoveshchenskaya A, Oorschot VM, Brankatschk B, Klumperman J, Freeze HH, Mayinger P. 2010. Spatial regulation of Golgi phosphatidylinositol-4-phosphate is required for enzyme localization and glycosylation fidelity. Traffic 11: 1180-1190.

Chiu VK, Bivona T, Hach A, Sajous JB, Silletti J, Wiener H, Johnson RLII, Cox AD, Philips MR. 2002. Ras signalling on the endoplasmic reticulum and the Golgi. Nat Cell Biol 4: 343-350.

D’Angelo G, Polishchuk E, Di Tullio G, Santoro M, Di Campli A, Godi A, West G, Bielawski J, Chuang CC, van der Spoel AC, et al. 2007. Glycosphingolipid synthesis requires FAPP2 transfer of glucosylceramide. Nature 449: $62-67$.

Dai Q, Choy E, Chiu V, Romano J, Slivka SR, Steitz SA, Michaelis S, Philips MR. 1998. Mammalian prenylcysteine carboxyl methyltransferase is in the endoplasmic reticulum. J Biol Chem 273: 15030-15034.

De Barry J, Janoshazi A, Dupont JL, Procksch O, ChasserotGolaz S, Jeromin A, Vitale N. 2006. Functional implication of neuronal calcium sensor- 1 and phosphoinositol 4-kinase- $\beta$ interaction in regulated exocytosis of PC12 cells. J Biol Chem 281: 18098-18111.

De Matteis MA, Di Campli A, Godi A. 2005. The role of the phosphoinositides at the Golgi complex. Biochim Biophys Acta 1744: 396-405.

Demmel L, Beck M, Klose C, Schlaitz AL, Gloor Y, Hsu PP, Havlis J, Shevchenko A, Krause E, Kalaidzidis Y, et al. 2008a. Nucleocytoplasmic shuttling of the Golgi phosphatidylinositol 4-kinase pik1 is regulated by 14-3-3 proteins and coordinates Golgi function with cell growth. Mol Biol Cell 19: 1046-1061.

Demmel L, Gravert M, Ercan E, Habermann B, MullerReichert T, Kukhtina V, Haucke V, Baust T, Sohrmann M, Kalaidzidis Y, et al. 2008b. The clathrin adaptor Gga2p is a phosphatidylinositol 4-phosphate effector at the Golgi exit. Mol Biol Cell 19: 1991-2002.

Di Paolo G, De Camilli P. 2006. Phosphoinositides in cell regulation and membrane dynamics. Nature 443: 651-657.

Diaz Anel AM, Malhotra V. 2005. PKCeta is required for $\beta 1 \gamma 2 / \beta 3 \gamma 2-$ and PKD-mediated transport to the cell surface and the organization of the Golgi apparatus. J Cell Biol 169: 83-91.

Dippold HC, Ng MM, Farber-Katz SE, Lee SK, Kerr ML, Peterman MC, Sim R, Wiharto PA, Galbraith KA, Madhavarapu S, et al. 2009. GOLPH3 bridges phosphatidylinositol-4- phosphate and actomyosin to stretch and shape the Golgi to promote budding. Cell 139: 337-351.

Edamatsu H, Satoh T, Kataoka T. 2006. Ras and Rap1 activation of PLCepsilon lipase activity. Methods Enzymol 407: 99-107.

Fan F, Feng L, He J, Wang X, Jiang X, Zhang Y, Wang Z, Chen Y. 2008. RKTG sequesters B-Raf to the Golgi apparatus and inhibits the proliferation and tumorigenicity of human malignant melanoma cells. Carcinogenesis 29: $1157-1163$.

Farhan H, Wendeler MW, Mitrovic S, Fava E, Silberberg Y, Sharan R, Zerial M, Hauri HP. 2010. MAPK signaling to the early secretory pathway revealed by kinase/phosphatase functional screening. J Cell Biol 189: 997-1011.

Faulhammer F, Kanjilal-Kolar S, Knodler A, Lo J, Lee Y, Konrad G, Mayinger P. 2007. Growth control of Golgi phosphoinositides by reciprocal localization of sacl lipid phosphatase and pik1 4-kinase. Traffic 8: 1554-1567.

Faulhammer F, Konrad G, Brankatschk B, Tahirovic S, Knodler A, Mayinger P. 2005. Cell growth-dependent coordination of lipid signaling and glycosylation is 
P. Mayinger

mediated by interactions between Sac1p and Dpm1p. J Cell Biol 168: 185-191.

Feng L, Xie X, Ding Q, Luo X, He J, Fan F, Liu W, Wang Z, Chen Y. 2007. Spatial regulation of Raf kinase signaling by RKTG. Proc Natl Acad Sci 104: 14348-14353.

Fivaz M, Meyer T. 2005. Reversible intracellular translocation of KRas but not HRas in hippocampal neurons regulated by $\mathrm{Ca}^{2+} /$ calmodulin. J Cell Biol 170: 429-441.

Fuchs YF, Eisler SA, Link G, Schlicker O, Bunt G, Pfizenmaier K, Hausser A. 2009. A Golgi PKD activity reporter reveals a crucial role of PKD in nocodazole-induced Golgi dispersal. Traffic 10: 858-867.

Fugmann T, Hausser A, Schoffler P, Schmid S, Pfizenmaier K, Olayioye MA. 2007. Regulation of secretory transport by protein kinase $\mathrm{D}$-mediated phosphorylation of the ceramide transfer protein. J Cell Biol 178: 15-22.

Godi A, Campli AD, Konstantakopoulos A, Tullio GD, Alessi DR, Kular GS, Daniele T, Marra P, Lucocq JM, Matteis MA. 2004. FAPPs control Golgi-to-cell-surface membrane traffic by binding to ARF and PtdIns(4)P. Nat Cell Biol 6: 393-404.

Godi A, Pertile P, Meyers R, Marra P, Di Tullio G, Iurisci C, Luini A, Corda D, De Matteis MA. 1999. ARF mediates recruitment of PtdIns-4-OH kinase- $\beta$ and stimulates synthesis of PtdIns(4,5)P2 on the Golgi complex. Nat Cell Biol 1: 280-287.

Goodwin JS, Drake KR, Rogers C, Wright L, LippincottSchwartz J, Philips MR, Kenworthy AK. 2005. Depalmitoylated Ras traffics to and from the Golgi complex via a nonvesicular pathway. J Cell Biol 170: 261-272.

Guo J, Wenk MR, Pellegrini L, Onofri F, Benfenati F, De Camilli P. 2003. Phosphatidylinositol 4-kinase type II $\alpha$ is responsible for the phosphatidylinositol 4-kinase activity associated with synaptic vesicles. Proc Natl Acad Sci 100: $3995-4000$.

Halter D, Neumann S, van Dijk SM, Wolthoorn J, de Maziere AM, Vieira OV, Mattjus P, Klumperman J, van Meer G, Sprong H. 2007. Pre- and post-Golgi translocation of glucosylceramide in glycosphingolipid synthesis. J Cell Biol 179: 101-115.

Hama H, Schnieders EA, Thorner J, Takemoto JY, DeWald DB. 1999. Direct involvement of phosphatidylinositol 4-phosphate in secretion in the yeast Saccharomyces cerevisiae. J Biol Chem 274: 34294-34300.

Han S, Lone MA, Schneiter R, Chang A. 2010. Orm1 and Orm2 are conserved endoplasmic reticulum membrane proteins regulating lipid homeostasis and protein quality control. Proc Natl Acad Sci 107: 5851-5856.

Hanada K, Kumagai K, Tomishige N, Kawano M. 2007. CERT and intracellular trafficking of ceramide. Biochim Biophys Acta 1771: 644-653.

Hanada K, Kumagai K, Yasuda S, Miura Y, Kawano M, Fukasawa M, Nishijima M. 2003. Molecular machinery for non-vesicular trafficking of ceramide. Nature 426: 803-809.

Hausser A, Link G, Hoene M, Russo C, Selchow O, Pfizenmaier K. 2006. Phospho-specific binding of 14-3-3 proteins to phosphatidylinositol 4-kinase III $\beta$ protects from dephosphorylation and stabilizes lipid kinase activity. J Cell Sci 119: 3613-3621.
Hausser A, Storz P, Martens S, Link G, Toker A, Pfizenmaier K. 2005. Protein kinase D regulates vesicular transport by phosphorylating and activating phosphatidylinositol-4 kinase III $\beta$ at the Golgi complex. Nat Cell Biol 7: $880-886$.

Haynes LP, Thomas GM, Burgoyne RD. 2005. Interaction of neuronal calcium sensor-1 and ADP-ribosylation factor 1 allows bidirectional control of phosphatidylinositol 4-kinase $\beta$ and trans-Golgi network-plasma membrane traffic. J Biol Chem 280: 6047-6054.

He H, Dai F, Yu L, She X, Zhao Y, Jiang J, Chen X, Zhao S. 2002. Identification and characterization of nine novel human small GTPases showing variable expressions in liver cancer tissues. Gene Expr 10: 231-242.

Heldwein EE, Macia E, Wang J, Yin HL, Kirchhausen T, Harrison SC. 2004. Crystal structure of the clathrin adaptor protein 1 core. Proc Natl Acad Sci 101: 14108-14113.

Hendricks KB, Wang BQ, Schnieders EA, Thorner J. 1999. Yeast homologue of neuronal frequenin is a regulator of phosphatidylinositol-4-OH kinase. Nat Cell Biol 1: 234-241.

Hsu NY, Ilnytska O, Belov G, Santiana M, Chen YH, Takvorian PM, Pau C, van der Schaar H, Kaushik-Basu $\mathrm{N}$, Balla $\mathrm{T}$, et al. 2010. Viral reorganization of the secretory pathway generates distinct organelles for RNA replication. Cell 141: 799-811.

Huttner IG, Strahl T, Osawa M, King DS, Ames JB, Thorner J. 2003. Molecular interactions of yeast frequenin (Frq1) with the phosphatidylinositol 4-kinase isoform, Pik1. J Biol Chem 278: 4862-4874.

Ibiza S, Perez-Rodriguez A, Ortega A, Martinez-Ruiz A, Barreiro O, Garcia-Dominguez CA, Victor VM, Esplugues JV, Rojas JM, Sanchez-Madrid F, et al. 2008. Endothelial nitric oxide synthase regulates N-Ras activation on the Golgi complex of antigen-stimulated T cells. Proc Natl Acad Sci 105: 10507-10512.

Kajimoto T, Ohmori S, Shirai Y, Sakai N, Saito N. 2001. Subtype-specific translocation of the $\delta$ subtype of protein kinase $\mathrm{C}$ and its activation by tyrosine phosphorylation induced by ceramide in HeLa cells. Mol Cell Biol 21: 1769-1783.

Kajimoto T, Shirai Y, Sakai N, Yamamoto T, Matsuzaki H, Kikkawa U, Saito N. 2004. Ceramide-induced apoptosis by translocation, phosphorylation, and activation of protein kinase $\mathrm{C} \delta$ in the Golgi complex. J Biol Chem 279: $12668-12676$.

Kawano M, Kumagai K, Nishijima M, Hanada K. 2006. Efficient trafficking of ceramide from the endoplasmic reticulum to the Golgi apparatus requires a VAMPassociated protein-interacting FFAT motif of CERT. $J$ Biol Chem 281: 30279-30288.

Kozminski KG, Alfaro G, Dighe S, Beh CT. 2006. Homologues of oxysterol-binding proteins affect $\mathrm{Cdc} 42 \mathrm{p}$ - and Rholp-mediated cell polarization in Saccharomyces cerevisiae. Traffic 7: 1224-1242.

Lenoir M, Coskun U, Grzybek M, Cao X, Buschhorn SB, James J, Simons K, Overduin M. 2010. Structural basis of wedging the Golgi membrane by FAPP pleckstrin homology domains. EMBO Rep 11: 279-284.

Lev S, Ben Halevy D, Peretti D, Dahan N. 2008. The VAP protein family: from cellular functions to motor neuron disease. Trends Cell Biol 18: 282-290. 
Li H, Adamik R, Pacheco-Rodriguez G, Moss J, Vaughan M. 2003. Protein kinase A-anchoring (AKAP) domains in brefeldin A-inhibited guanine nucleotide-exchange protein 2 (BIG2). Proc Natl Acad Sci 100: 1627-1632.

Li X, Rivas MP, Fang M, Marchena J, Mehrotra B, Chaudhary A, Feng L, Prestwich GD, Bankaitis VA. 2002. Analysis of oxysterol binding protein homologue Keslp function in regulation of Sec14p-dependent protein transport from the yeast Golgi complex. J Cell Biol 157: 63-77.

Maeda Y, Beznoussenko GV, Van Lint J, Mironov AA, Malhotra V. 2001. Recruitment of protein kinase D to the trans-Golgi network via the first cysteine-rich domain. Embo J 20: 5982-5990.

Malhotra V, Campelo F. 2010. PKD regulates membrane fission to generate TGN to cell surface transport carriers. Cold Spring Harb Perspect Biol doi:10.1101/ cshperspect.a005280.

Martin ME, Hidalgo J, Rosa JL, Crottet P, Velasco A. 2000. Effect of protein kinase A activity on the association of ADP-ribosylation factor 1 to golgi membranes. J Biol Chem 275: 19050-19059.

Martin ME, Hidalgo J, Vega FM, Velasco A. 1999. Trimeric G proteins modulate the dynamic interaction of PKAII with the Golgi complex. J Cell Sci 112: 3869-3878.

Mavillard F, Hidalgo J, Megias D, Levitsky KL, Velasco A. 2010. PKA-mediated Golgi remodeling during cAMP signal transmission. Traffic 11: 90-109.

Mayinger P. 2009. Regulation of Golgi function via phosphoinositide lipids. Semin Cell Dev Biol 20: 793-800.

Minogue S, Waugh MG, De Matteis MA, Stephens DJ Berditchevski F, Hsuan JJ. 2006. Phosphatidylinositol 4-kinase is required for endosomal trafficking and degradation of the EGF receptor. J Cell Sci 119: 571-581.

Mitin NY, Ramocki MB, Zullo AJ, Der CJ, Konieczny SF, Taparowsky EJ. 2004. Identification and characterization of rain, a novel Ras-interacting protein with a unique subcellular localization. J Biol Chem 279: 22353-22361.

Mizuno-Yamasaki E, Medkova M, Coleman J, Novick P. 2010. Phosphatidylinositol 4-phosphate controls both membrane recruitment and a regulatory switch of the Rab GEF Sec2p. Dev Cell 18: 828-840.

Muniz M, Alonso M, Hidalgo J, Velasco A. 1996. A regulatory role for cAMP-dependent protein kinase in protein traffic along the exocytic route. J Biol Chem 271: 30935-30941.

Muniz M, Martin ME, Hidalgo J, Velasco A. 1997. Protein kinase A activity is required for the budding of constitutive transport vesicles from the trans-Golgi network. Proc Natl Acad Sci 94: 14461-14466.

Ngo M, Ridgway ND. 2009. Oxysterol binding proteinrelated Protein 9 (ORP9) is a cholesterol transfer protein that regulates Golgi structure and function. Mol Biol Cell 20: $1388-1399$.

Nigg EA, Hilz H, Eppenberger HM, Dutly F. 1985. Rapid and reversible translocation of the catalytic subunit of cAMP-dependent protein kinase type II from the Golgi complex to the nucleus. EMBO Journal 4: 2801-2806.

Pelham HR. 1991. Recycling of proteins between the endoplasmic reticulum and Golgi complex. Curr Opin Cell Biol 3: 585-591.
Peretti D, Dahan N, Shimoni E, Hirschberg K, Lev S. 2008. Coordinated lipid transfer between the endoplasmic reticulum and the Golgi complex requires the VAP proteins and is essential for Golgi-mediated transport. Mol Biol Cell 19: 3871-3884.

Perez de Castro I, Bivona TG, Philips MR, Pellicer A. 2004. Ras activation in Jurkat $\mathrm{T}$ cells following low-grade stimulation of the T-cell receptor is specific to N-Ras and occurs only on the Golgi apparatus. Mol Cell Biol 24: 3485-3496.

Pulvirenti T, Giannotta M, Capestrano M, Capitani M, Pisanu A, Polishchuk RS, San Pietro E, Beznoussenko GV, Mironov AA, Turacchio G, et al. 2008. A trafficactivated Golgi-based signalling circuit coordinates the secretory pathway. Nat Cell Biol 10: 912-922.

Quatela SE, Philips MR. 2006. Ras signaling on the Golgi. Curr Opin Cell Biol 18: 162-167.

Riebeling C, Morris AJ, Shields D. 2009. Phospholipase D in the Golgi apparatus. Biochim Biophys Acta 1791: 876-880.

Rocks O, Peyker A, Kahms M, Verveer PJ, Koerner C, Lumbierres M, Kuhlmann J, Waldmann H, Wittinghofer A, Bastiaens PI. 2005. An acylation cycle regulates localization and activity of palmitoylated Ras isoforms. Science 307: 1746-1752.

Roy A, Levine TP. 2004. Multiple pools of phosphatidylinositol 4-phosphate detected using the pleckstrin homology domain of Osh2p. J Biol Chem 279: 44683-44689.

Saito S, Matsui H, Kawano M, Kumagai K, Tomishige N, Hanada K, Echigo S, Tamura S, Kobayashi T. 2008. Protein phosphatase 2Cepsilon is an endoplasmic reticulum integral membrane protein that dephosphorylates the ceramide transport protein CERT to enhance its association with organelle membranes. J Biol Chem 283: 6584-6593.

Sallese M, Giannotta M, Luini A. 2009. Coordination of the secretory compartments via inter-organelle signalling. Semin Cell Dev Biol 20: 801-809.

Schmidt WK, Tam A, Fujimura-Kamada K, Michaelis S. 1998. Endoplasmic reticulum membrane localization of Rcelp and Ste24p, yeast proteases involved in carboxylterminal CAAX protein processing and amino-terminal a-factor cleavage. Proc Natl Acad Sci 95: 11175-11180.

Schmitz KR, Liu J, Li S, Setty TG, Wood CS, Burd CG, Ferguson KM. 2008. Golgi localization of glycosyltransferases requires a Vps74p oligomer. Dev Cell 14: $523-534$.

Schorr M, Then A, Tahirovic S, Hug N, Mayinger P. 2001. The phosphoinositide phosphatase Saclp controls trafficking of the yeast Chs3p chitin synthase. Curr Biol 11: 1421-1426.

Schultz A, Jonsson JI, Larsson C. 2003. The regulatory domain of protein kinase $\mathrm{C} \theta$ localises to the Golgi complex and induces apoptosis in neuroblastoma and Jurkat cells. Cell Death Differ 10: 662-675.

Schultz A, Ling M, Larsson C. 2004. Identification of an amino acid residue in the protein kinase $\mathrm{C} \mathrm{Clb}$ domain crucial for its localization to the Golgi network. J Biol Chem 279: 31750-31760.

Schulz TA, Choi MG, Raychaudhuri S, Mears JA, Ghirlando R, Hinshaw JE, Prinz WA. 2009. Lipid-regulated sterol transfer between closely apposed membranes by 
P. Mayinger

oxysterol-binding protein homologues. J Cell Biol 187: 889-903.

Scott KL, Kabbarah O, Liang MC, Ivanova E, Anagnostou V, Wu J, Dhakal S, Wu M, Chen S, Feinberg T, et al. 2009. GOLPH3 modulates mTOR signalling and rapamycin sensitivity in cancer. Nature 459: 1085-1090.

Shanks RA, Steadman BT, Schmidt PH, Goldenring JR. 2002. AKAP350 at the Golgi apparatus. I. Identification of a distinct Golgi apparatus targeting motif in AKAP350. J Biol Chem 277: 40967-40972.

Sonoda H, Okada T, Jahangeer S, Nakamura S. 2007. Requirement of phospholipase D for ilimaquinoneinduced Golgi membrane fragmentation. J Biol Chem 282: 34085-34092.

Strahl T, Hama H, DeWald DB, Thorner J. 2005. Yeast phosphatidylinositol 4-kinase, Pik1, has essential roles at the Golgi and in the nucleus. J Cell Biol 171: 967-979.

Swarthout JT, Lobo S, Farh L, Croke MR, Greentree WK, Deschenes RJ, Linder ME. 2005. DHHC9 and GCP16 constitute a human protein fatty acyltransferase with specificity for H- and N-Ras. J Biol Chem 280: 31141-31148.

Toth B, Balla A, Ma H, Knight ZA, Shokat KM, Balla T. 2006. Phosphatidylinositol 4-kinase III $\beta$ regulates the transport of ceramide between the endoplasmic reticulum and Golgi. J Biol Chem 281: 36369-36377.

Tsai FM, Shyu RY, Jiang SY. 2006. RIG1 inhibits the Ras/ mitogen-activated protein kinase pathway by suppressing the activation of Ras. Cell Signal 18: 349-358.

Tu L, Tai WC, Chen L, Banfield DK. 2008. Signal-mediated dynamic retention of glycosyltransferases in the Golgi. Science 321: 404-407.

Varnai P, Balla T. 2008. Live cell imaging of phosphoinositides with expressed inositide binding protein domains. Methods 46: 167-176.

Vicinanza M, D’Angelo G, Di Campli A, De Matteis MA. 2008. Function and dysfunction of the PI system in membrane trafficking. Embo J 27: 2457-2470.

Vieira OV, Verkade P, Manninen A, Simons K. 2005. FAPP2 is involved in the transport of apical cargo in polarized MDCK cells. J Cell Biol 170: 521-526.

Villani M, Subathra M, Im YB, Choi Y, Signorelli P, Del Poeta M, Luberto C. 2008. Sphingomyelin synthases regulate production of diacylglycerol at the Golgi. Biochem J 414: $31-41$.

Walch-Solimena C, Novick P. 1999. The yeast phosphatidylinositol-4-OH kinase pik1 regulates secretion at the Golgi. Nat Cell Biol 1: 523-525.

Wang Y, Seemann J. 2011. Golgi biogenesis. Cold Spring Harb Perspect Biol doi:10.1101/cshperspect.a005330.

Wang J, Sun HQ, Macia E, Kirchhausen T, Watson H, Bonifacino JS, Yin HL. 2007. PI4P promotes the recruitment of the GGA adaptor proteins to the trans-Golgi network and regulates their recognition of the ubiquitin sorting signal. Mol Biol Cell 18: 2646-2655.

Wang YJ, Wang J, Sun HQ, Martinez M, Sun YX, Macia E, Kirchhausen T, Albanesi JP, Roth MG, Yin HL. 2003. Phosphatidylinositol 4 phosphate regulates targeting of clathrin adaptor AP-1 complexes to the Golgi. Cell 114: 299-310.

Weixel KM, Blumental-Perry A, Watkins SC, Aridor M, Weisz OA. 2005. Distinct Golgi populations of phosphatidylinositol 4-phosphate regulated by phosphatidylinositol 4-kinases. J Biol Chem 280: 10501-10508.

Weller SG, Capitani M, Cao H, Micaroni M, Luini A, Sallese M, McNiven MA. 2010. Src kinase regulates the integrity and function of the Golgi apparatus via activation of dynamin 2. Proc Natl Acad Sci 107: 5863-5868.

Wojtal KA, de Vries E, Hoekstra D, van Ijzendoorn SC. 2006. Efficient trafficking of MDR1/P-glycoprotein to apical canalicular plasma membranes in HepG2 cells requires PKA-RII $\alpha$ anchoring and glucosylceramide. Mol Biol Cell 17: 3638-3650.

Wood CS, Schmitz KR, Bessman NJ, Setty TG, Ferguson KM, Burd CG. 2009. PtdIns4P recognition by Vps74/ GOLPH3 links PtdIns 4-kinase signaling to retrograde Golgi trafficking. J Cell Biol 187: 967-975.

Yamaji T, Kumagai K, Tomishige N, Hanada K. 2008. Two sphingolipid transfer proteins, CERT and FAPP2: Their roles in sphingolipid metabolism. IUBMB Life 60: $511-518$.

Yasuda T, Kurosaki T. 2008. Regulation of lymphocyte fate by Ras/ERK signals. Cell Cycle 7: 3634-3640.

Yasuda S, Kitagawa H, Ueno M, Ishitani H, Fukasawa M, Nishijima M, Kobayashi S, Hanada K. 2001. A novel inhibitor of ceramide trafficking from the endoplasmic reticulum to the site of sphingomyelin synthesis. $J$ Biol Chem 276: 43994-44002.

Yui N, Okutsu R, Sohara E, Rai T, Ohta A, Noda Y, Sasaki S, Uchida S. 2009. FAPP2 is required for aquaporin-2 apical sorting at trans-Golgi network in polarized MDCK cells. Am J Physiol Cell Physiol 297: C1389-C1396.

Zegers MM, Hoekstra D. 1997. Sphingolipid transport to the apical plasma membrane domain in human hepatoma cells is controlled by PKC and PKA activity: A correlation with cell polarity in HepG2 cells. J Cell Biol 138: 307-321.

Zhang M, Xia H, Li X, Wang X, Dong Y, Zhang T, Yu H. 2010. C1 domain mediates CalDAGIII localization to the Golgi. Mol Biol Rep 37: 3481-3485.

Zhao X, Varnai P, Tuymetova G, Balla A, Toth ZE, Oker-Blom C, Roder J, Jeromin A, Balla T. 2001. Interaction of neuronal calcium sensor-1 (NCS-1) with phosphatidylinositol 4-kinase $\beta$ stimulates lipid kinase activity and affects membrane trafficking in COS-7 cells. J Biol Chem 276: 40183-40189. 


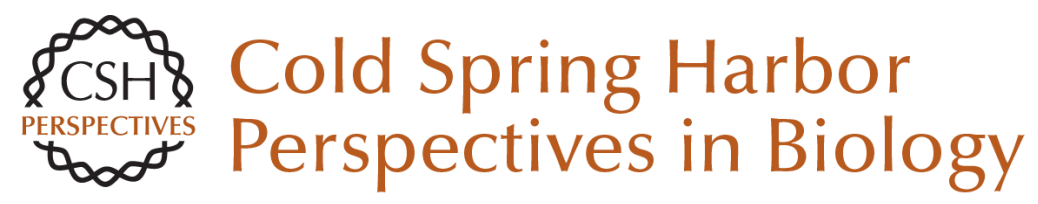

\section{Signaling at the Golgi}

Peter Mayinger

Cold Spring Harb Perspect Biol 2011; doi: 10.1101/cshperspect.a005314 originally published online March 30, 2011

\section{Subject Collection The Golgi}

Structure of Golgi Transport Proteins

Daniel Kümmel and Karin M. Reinisch

\section{Golgi Biogenesis}

Yanzhuang Wang and Joachim Seemann

Golgi Glycosylation and Human Inherited

Diseases

Hudson H. Freeze and Bobby G. Ng

Models for Golgi Traffic: A Critical Assessment Benjamin S. Glick and Alberto Luini

\section{Architecture of the Mammalian Golgi} Judith Klumperman

Evolution and Diversity of the Golgi Mary J. Klute, Paul Melançon and Joel B. Dacks

Evolutionary Forces Shaping the Golgi

Glycosylation Machinery: Why Cell Surface

Glycans Are Universal to Living Cells Ajit Varki

Golgi Positioning

Smita Yadav and Adam D. Linstedt
Golgi and Related Vesicle Proteomics: Simplify to Identify Joan Gannon, John J.M. Bergeron and Tommy Nilsson

Organization of SNAREs within the Golgi Stack Jörg Malsam and Thomas H. Söllner

Golgi during Development Weimin Zhong

Entry and Exit Mechanisms at the cis-Face of the Golgi Complex Andrés Lorente-Rodríguez and Charles Barlowe

COPI Budding within the Golgi Stack Vincent Popoff, Frank Adolf, Britta Brügger, et al.

Mechanisms of Protein Retention in the Golgi David K. Banfield

The Golgin Coiled-Coil Proteins of the Golgi

Apparatus Sean Munro

Signaling at the Golgi Peter Mayinger

For additional articles in this collection, see http://cshperspectives.cshlp.org/cgi/collection/

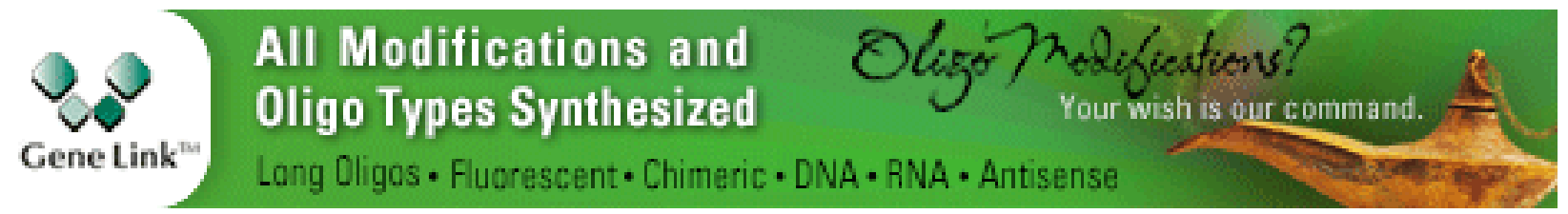

Copyright @ 2011 Cold Spring Harbor Laboratory Press; all rights reserved 